\title{
CYP2J2 and EETs Protect Against Lung Ischemia/Reperfusion Injury via Anti- Inflammatory Effects in Vivo and in Vitro
}

\author{
Wenshu Chen ${ }^{a, b}$ Shijiang Yang ${ }^{c}$ Wei Ping ${ }^{a}$ Xiangning Fu ${ }^{a}$ Qinzi Xu ${ }^{a}$ \\ Jianing Wang ${ }^{a}$ \\ aDepartment of Thoracic Surgery, Tongji Hospital, Tongji Medical College, Huazhong University of \\ Science and Technology, Wuhan, bDepartment of Thoracic Surgery, Fujian Provincial Hospital, Provincial \\ Clinic College of Fujian Medical University, Fuzhou, 'Department of Thoracic Surgery, the Central \\ Hospital of Wuhan, Wuhan, China
}

\section{Key Words}

Cytochrome P450 epoxygenase 2J2 • Epoxyeicosatrienoic acid • Lung ischemia/reperfusion injury $\cdot$ Inflammation

\begin{abstract}
Background: Injurious inflammatory response is critical to the development of lung ischemia/ reperfusion injury (LIRI). The cytochrome P450 epoxygenase 2J2 (CYP2J2) metabolizes arachidonic acid to epoxyeicosatrienoic acids (EETs), which exert an anti-inflammatory effect on the cardiovascular system. We therefore hypothesized that CYP2J2 overexpression and pretreatment with exogenous EETs may have the potential to reduce LIRI. Methods: A rat model was used to mimic the condition of LIRI by clamping the left pulmonary hilum for 60 minutes, followed by reperfusion for 2 hours. Moreover, we developed a cell model using human pulmonary artery endothelial cells by anoxia for 8 hours, followed by reoxygenation for 16 hours to determine the anti-inflammatory effect and mechanism of CYP2J2 overexpression and exogenous 11,12-EET. Results: Lung ischemia/reperfusion increased lung wet/dry and lung weight/body weight ratios, protein concentration in bronchoalveolar lavage fluid and concentrations of pro-inflammatory mediators in serum, including IL-1 $\beta$, IL-8, TNF- $\alpha$, sP- and sE-selectin, and decreased concentration of anti-inflammatory mediator IL-10. Ischemia/ reperfusion also leaded to pulmonary edema and inflammation under light microscopy. Furthermore, activation of NF-kB p65 and degradation of IkB $\alpha$ were remarkably increased in ischemia/reperfusion lung tissues. While CYP2J2 overexpression significantly inhibited the above effects $(p<0.05)$. In vitro data further confirmed the anti-inflammatory effect of CYP2J2 overexpression and 11,12-EET, an effect that may probably be mediated by PPARY activation. Conclusion: CYP2J2 overexpression and administration of exogenous EETs can protect against LIRI via anti-inflammatory effects. This can be a novel potential strategy for prevention and treatment of LIRI.

W. Chen and S. Yang contributed equally to this work.

\begin{tabular}{ll}
\hline Jianing Wang, MD, PhD & Department of Thoracic Surgery, Tongji Hospital, Tongji Medical College, Huazhong \\
& University of Science and Technology; 1095 Jiefang Ave., Wuhan 430030, (P.R. China) \\
& Tel./Fax +86-27-8366-5291, E-Mail wjn4002@aliyun.com
\end{tabular}
\end{abstract}




\section{Cellular Physiology Cell Physiol Biochem 2015;35:2043-2054

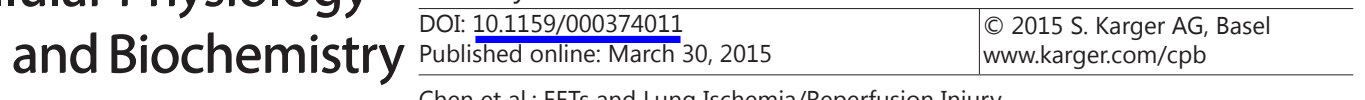

\section{Introduction}

Ischemia/reperfusion (IR)-induced acute lung injury (ALI) remains a life-threatening but inevitable complication in various clinical conditions, such as lung transplantation, cardiopulmonary bypass, bronchovascular sleeve resection and reconstruction, resuscitation for circulatory arrest, and pulmonary embolism [1]. Re-establishment of perfusion in the ischemic lung is a double-edged sword, it is not only essential to restore tissue function, but also triggers a complex cascade of events leading to lung injury, characterized by increased pulmonary vascular permeability, pulmonary edema, microvascular damage, release of inflammatory mediators, and production of reactive oxygen species $[2,3]$. The underlying pathophysiological mechanisms during the process of LIRI are complex and multifactorial. Over the past few decades, although various therapeutic strategies have been developed to treat or attenuate LIRI, their clinical efficacy is limited [2]. Further investigation is needed to explore novel treatments for LIRI.

Cytochrome P450 epoxygenase 2J2 (CYP2J2), which is abundantly expressed in vascular endothelial cells and cardiac myocytes [4], metabolizes arachidonic acid to four epoxyeicosatrienoic acids (5,6-, 8,9-, 11,12-, and 14,15-EETs) [5]. EETs exert a variety of biological functions in normal and pathophysiological conditions, including antiinflammatory [4, 5], anti-apoptotic [6], anti-fibrotic [7], and anti-oxidant [8]. Furthermore, accumulating evidence demonstrates that EETs protect against global cerebral and myocardium IR injury $[9,10]$. However, little is known about the effect and mechanism of CYP2J2 and EETs during LIRI. In previous study, we have confirmed that gene delivery leads to CYP2J2 overexpression in lung tissues of transgenic rats, especially in pulmonary artery endothelial cells [11], which are critical mediators in acute LIRI [12]. In the present study, we will further investigate the relationship between CYP2J2 overexpression and LIRI.

It is well documented that injurious inflammatory responses play a critical role during LIRI. EETs exert anti-inflammatory effects via activation of peroxisome proliferatoractivated receptor $\gamma$ (PPAR $\gamma$ ) $[5,6]$. It has also been reported that glutamine protects against IR-induced ALI in isolated rat lungs via inhibition of nuclear factor (NF)- $\kappa B$ activation [13]. In addition, PPAR $\gamma$ agonists have been shown to inhibit the activation of NF-kB [14]. We therefore hypothesized that increased circulating levels of EETs by CYP2J2 overexpression in vivo may protect against LIRI via PPAR $\gamma$ activation.

\section{Material and Methods}

\section{Construction of gene delivery vector}

The CYP2J2 cDNA or green fluorescent protein (GFP) cDNA was respectively subcloned into the pcDNA3.1 plasmid as previously described [4]. The vectors were purified using the QIAGEN Plasmid Maxi Kit (QIAGEN, Inc., Chatsworth, CA) according to the manufacturer's instructions.

\section{Animal model and gene delivery protocols}

All animal experimental protocols complied with the National Institutes of Health Guidelines for the Care and Use of Laboratory Animals and were approved by the Institutional Animal Research Committee of Tongji Medical College. All surgeries were performed under pentobarbital sodium anesthesia via intraperitoneal injection.

Male Wistar rats weighing 280 to $350 \mathrm{~g}$ were obtained from the Experimental Animal Center of Hubei Province, transported in sterile conditions and housed at $25^{\circ} \mathrm{C}$ with 12 hours light/dark cycles. After one week adaptation, animals were randomly assigned into five groups ( $\mathrm{n}=10$ in each group): Blank (blank control group), Sham (sham group), IR (lung ischemia/reperfusion group), IR+GFP (lung ischemia/reperfusion and pcDNA3.1-GFP gene transfection group), and IR+CYP2J2 (lung ischemia/reperfusion and pcDNA3.1-CYP2J2 gene transfection group). Animals from IR+GFP and IR+CYP2J2 groups received a volume of 


\section{Cellular Physiology Cell Physiol Biochem 2015;35:2043-2054 \begin{tabular}{l|l}
\hline and Biochemistry 10.1159/000374011 & $\begin{array}{l}\text { C) } 2015 \text { S. Karger AG, Basel } \\
\text { www.karger.com/cpb }\end{array}$ \\
\cline { 2 - 3 }
\end{tabular} \\ Chen et al.: EETs and Lung Ischemia/Reperfusion Injury}

plasmid at a dose of $3 \mathrm{mg} / \mathrm{kg}$ body weight via the tail vein, once a week for two consecutive weeks. Animals from the other three groups received an equal volume of saline at the same time.

One week after the second injection, animals from the IR groups received a left anterolateral thoracotomy via the fifth intercostal space. Heparin was injected into the right ventricle at a dose of $100 \mathrm{IU} / \mathrm{kg}$ to prevent thrombosis. 5 minutes later, the left pulmonary hilum, including the main bronchus, bronchial arteries, pulmonary artery and vein, was dissected and occluded by a noninvasive artery clamp. After 1 hour of complete ischemia, the artery clamp was removed and the left lung was allowed to ventilate and reperfuse for an additional 2 hours. Time-matched sham group rats underwent identical surgical procedures, except for ischemia/reperfusion. The Blank group did not undergo surgery. The whole procedure was conducted under anesthesia and mechanical ventilation [13].

During the entire procedure, rats were placed on a warm bed and under a heating light to keep warm. Body temperature was monitored continuously and maintained at about 37 degrees. The wounds were kept covered with warm, saline-soaked gauze to prevent excessive fluid loss. Animals were sacrificed at the end of the experiment. Blood samples were obtained from the inferior vena cava just prior to sacrifice. The left lung was then removed for further analysis.

\section{Lung immunohistochemistry and histopathologic examination}

The expression of CYP2J2 protein in lung tissues was detected by immunohistochemical staining using a treptavidin/Peroxidase immunohistochemical kit (Fuzhou Maixin Biotechnology Co. Ltd, Fujian, China) according to the manufacturer's instruction [15]. The result was examined by two independent investigators in a double-blind fashion. At least three randomly selected fields $(\times 400)$ were assessed.

Immediately after the animals were sacrificed, the lungs were removed, inflated, and then divided into four parts. One part was put into a specimen basket and then fixed overnight in $10 \%$ formalin. In the present study, no pulmonary infarction was found. The lung tissues were processed using standard methods, as reported previously [16], and stained with hematoxylin and eosin. The samples were independently observed by two investigators in a double-blind fashion. At least 5 sections in each lung and 5 randomly selected high-power fields $(\times 200)$ for each section were evaluated. Based on the degree of pulmonary edema, hemorrhage and interstitial inflammatory cell infiltration, the lung injury was graded as follows: 0 , no evidence; 1 , mild injury; 2 , moderate injury; and 3 , severe injury $[17,18]$. In addition, it has been confirmed in our previous study that the expression of CYP2J2 protein was significantly increased in lung tissues of transgenic rats, especially in the cytoplasm of vascular endothelial cells [11].

Measurement of lung weight/body weight (LW/BW) and lung wet weight/dry weight $(W / D)$ ratio

At the end of the experiments, the left lung was taken out of the hilum and the wet weight was measured to calculate the LW/BW ratio. The sample was then put into an oven at $65^{\circ} \mathrm{C}$ for 72 hours to assess the wet/dry weight (W/D) ratio.

Protein concentration in bronchoalveolar lavage fluid

Immediately after the rat was sacrificed, the trachea was exposed and cannulated, accompanied with ligation of the right main bronchus. The left lung was then lavaged with $10 \mathrm{ml} / \mathrm{kg}$ of sterile saline and aspirated gently. The fluid was withdrawn 30 seconds later. The above procedure was repeated three times. Collected bronchoalveolar lavage fluid (BALF) was placed on ice followed by centrifugation at $3000 \mathrm{~g}$ for 10 minutes at $4^{\circ} \mathrm{C}$. The supernatant was collected and stored at $-20^{\circ} \mathrm{C}$ for subsequent analysis. The total protein concentration of the BALF supernatant was measured using the BCA Protein Assay Reagent Kit (Pierce, Rockford, IL), according to manufacturer's instruction. 


\section{Cellular Physiology Cell Physiol Biochem 2015;35:2043-2054

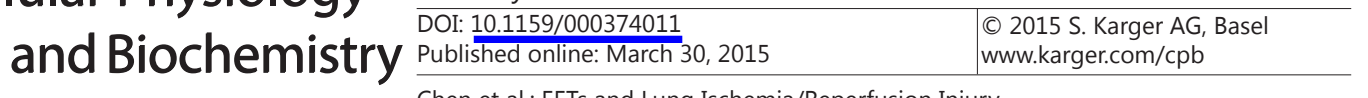

\section{Cell culture and gene transfection}

Human pulmonary artery endothelial cells (HPAECs) were purchased from ScienCell Research Laboratories (Carlsbad, CA, USA) and cultured according to the manufacturer's protocol. Cells were plated in 6-well plates, and transfected with pcDNA3.1-GFP or pcDNA3.1CYP2J2 after approximately 60\% confluence with Fugene HD Transfection Reagent (Roche Molecular Biochemicals, Indianapolis, IN). The transfection efficiency was detected by examining pcDNA3.1-GFP-transfected cells for GFP fluorescence under a fluorescence microscope (NIKON) and flow cytometric analysis. We have confirmed that the transfection efficiency was maintained at 35-60\% within 60 hours after transfection [11]. The expression of CYP2J2 was confirmed by immunoblotting.

\section{Induction of anoxia-reoxygenation in vitro and cell treatment}

The anoxia-reoxygenation (AR) model used is based on previous studies $[9,11,17]$. Briefly, cells in 6-well plates were grown to approximately $80 \%$ in a normoxic incubator (5\% CO2, 95\% air, $37^{\circ} \mathrm{C}, 95 \%$ humidity). Cells were then pretreated with GW9662 (PPAR $\gamma$ inhibitor, 1 umol/L) for 30 minutes before addition of EET (1umol/L) or vehicle, which were added 1 hour before AR. For the AR groups, culture plates were replaced with fresh endothelial cell medium (ScienCell Research Laboratories) without serum, and subjected to anoxia $\left(5 \% \mathrm{CO} 2,95 \% \mathrm{~N} 2,37^{\circ} \mathrm{C}\right)$ for 8 hours, followed by reoxygenation $(5 \% \mathrm{CO} 2,95 \%$ air $)$ for an additional 16 hours. Before reoxygenation, culture plates were replaced with complete endothelial cell medium. Normal control cells received the identical processes for 24 hours except for anoxia/reoxygenation.

Determination of inflammatory mediators in rat serum and in cell cultured media by ELISA

The concentrations of interleukin (IL)-1 $\beta$, IL-6, IL-8, IL-10, tumor necrosis factor (TNF)- $\alpha$, soluble P- and E-selectin (sP- and sE-selectin) in rat serum, and IL-1 $\beta$, IL-6, IL-10, intercellular adhesion molecule-1 (ICAM-1), and vascular endothelial adhesion molecule-1 (VCAM-1) in HPAECs cultured media were measured using enzyme-linked immunosorbent assay (ELISA) kits (NeoBioscience Technology Co., Ltd., Shenzhen, China), according to the manufacturer's instructions.

\section{Western Blot analysis}

Protein was extracted from lung tissues and cells as per the previous study [9]. Protein concentrations were determined using the Bradford method using BCA Protein Assay Reagent. Western Blots were performed as per the previous study [19]. $\beta$-actin was used as an internal reference for total protein or cytosolic protein determination, while lamin B1 was used for nuclear protein determination. Secondary antibodies against rabbit or mouse immunoglobulin (Ig) G conjugated to horseradish peroxidase were purchased from Jackson ImmunoResearch Laboratories (West Grove, PA, USA). Protein expression was quantified by densitometry and normalized to the internal reference.

\section{Statistical analysis}

All data were presented as mean \pm standard deviation (SD). Statistical differences between groups were evaluated by a one-way analysis of variance (ANOVA). $P<0.05$ was accepted as significant difference.

\section{Results}

Effects of CYP2J2 gene delivery on CYP2J2 protein expression in vivo and in vitro, and on circulating EETs level

One week after the second gene delivery, CYP2J2 protein was overexpressed in lung tissues of transgenic rats examined by Western Blot (Fig. 1A). CYP2J2 metabolizes 


\section{Cellular Physiology \\ Cell Physiol Biochem 2015;35:2043-2054

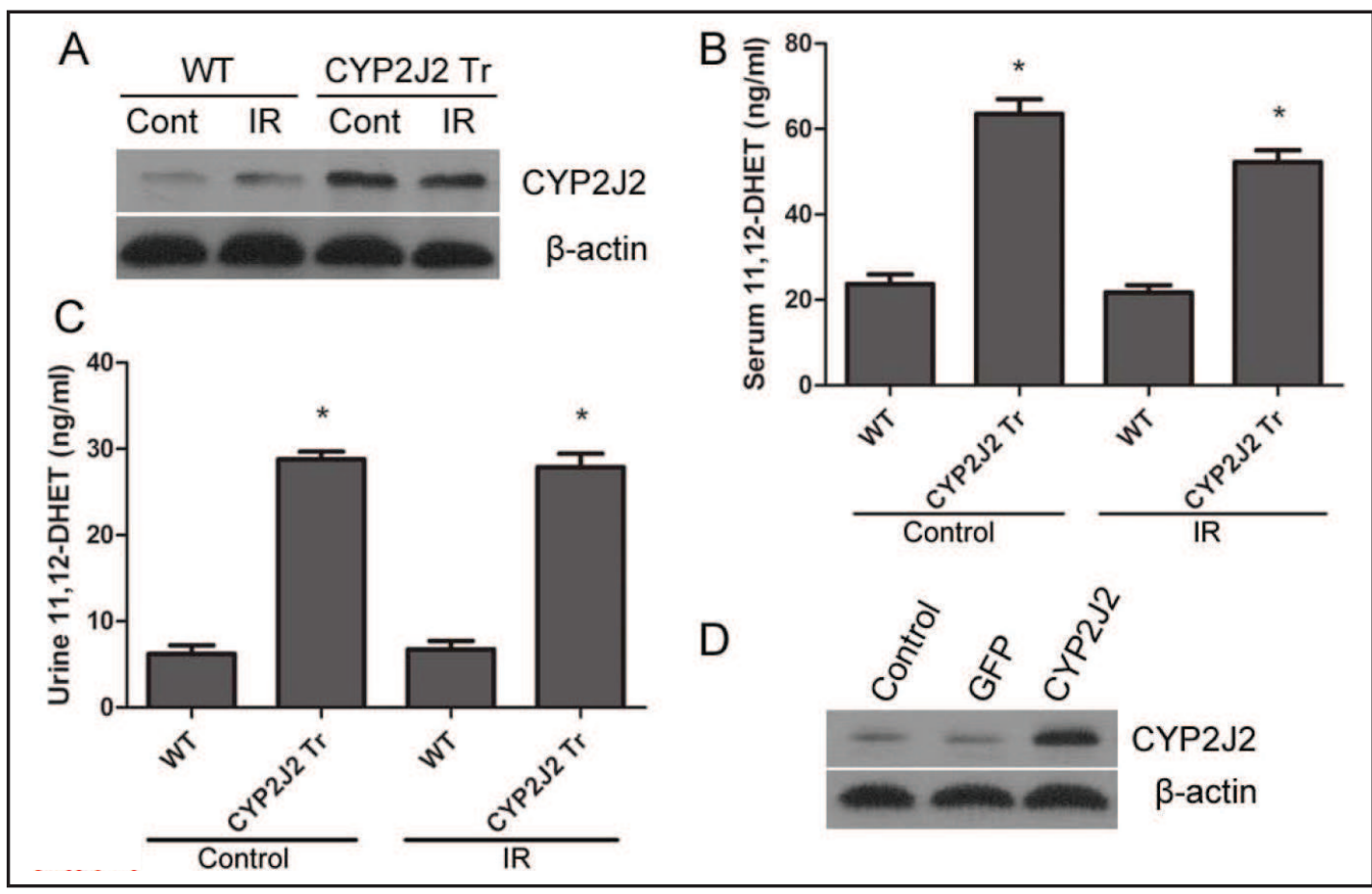

Fig. 1. Effects of pcDNA3.1-CYP2J2 delivery on CYP2J2 expression in vivo and in vitro, and on circulating EET synthesis. (A) CYP2J2 was overexpressed in lung tissue of transgenic rats compared to wild-type rats under both control and IR conditions. (B and C) 11,12-DHET levels were significantly higher in serum and urine of transgenic rats than in wild-type rats under both control and IR conditions. ( $\mathrm{n}=5, * p<0.05$ versus WT). (D) CYP2J2 protein was overexpressed after CYP2J2 gene delivery measured by Western Blot.

arachidonic acids to EETs, which can be oxidized to their corresponding DHETs with more stable biological activity. We therefore detected 11,12-DHET levels in rats, and found that CYP2J2 gene delivery resulted in a significant increase in both plasma and urine compared to wild-type rats ( $p<0.05$; Fig. 1B and C). In addition, CYP2J2 gene delivery led to CYP2J2 protein overexpresion in HPAECs (Fig. 1D).

\section{Effects of CYP2J2 overexpression on lung immunohistochemistry and histopathology}

As indicated in Fig. 2A, CYP2J2 protein expression was significantly increased in lung tissues of transgenic rats, especially in the cytoplasm of vascular endothelial cells and epithelial cells. The cytoplasm of CYP2J2 positive cells were stained brown in transgenic rats, as reported previously $[11,20]$.

Moreover, lung tissues in the Blank and Sham groups under light microscopy showed normal architecture and no inflammatory cell infiltration (Fig. 2B). In the IR groups, there was significant interstitial and perivascular edema, and inflammatory cell infiltration in interstitium and alveolar spaces, intra-alveolar fibrin deposition and hemorrhage. Alveolar septum was notably widened by edema, erythrocyte extravasation and fibrin deposition. However, CYP2J2 overexpression effectively extenuated pulmonary edema and inflammatory cell infiltration. The lung injury scores further confirmed the protective role of CYP2J2 overexpression against acute IR injury (Fig. 2C).

\section{Effects of CYP2J2 overexpression on lung $W / D$ and $L W / B W$ ratios}

As depicted in Fig. 3, the lung W/D and LW/BW ratios significantly increased following lung IR compared with control groups without IR, while the effect was reversed by CYP2J2 overexpression. 


\section{Cellular Physiology Cell Physiol Biochem 2015;35:2043-2054

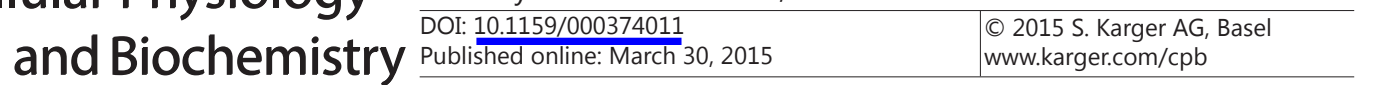

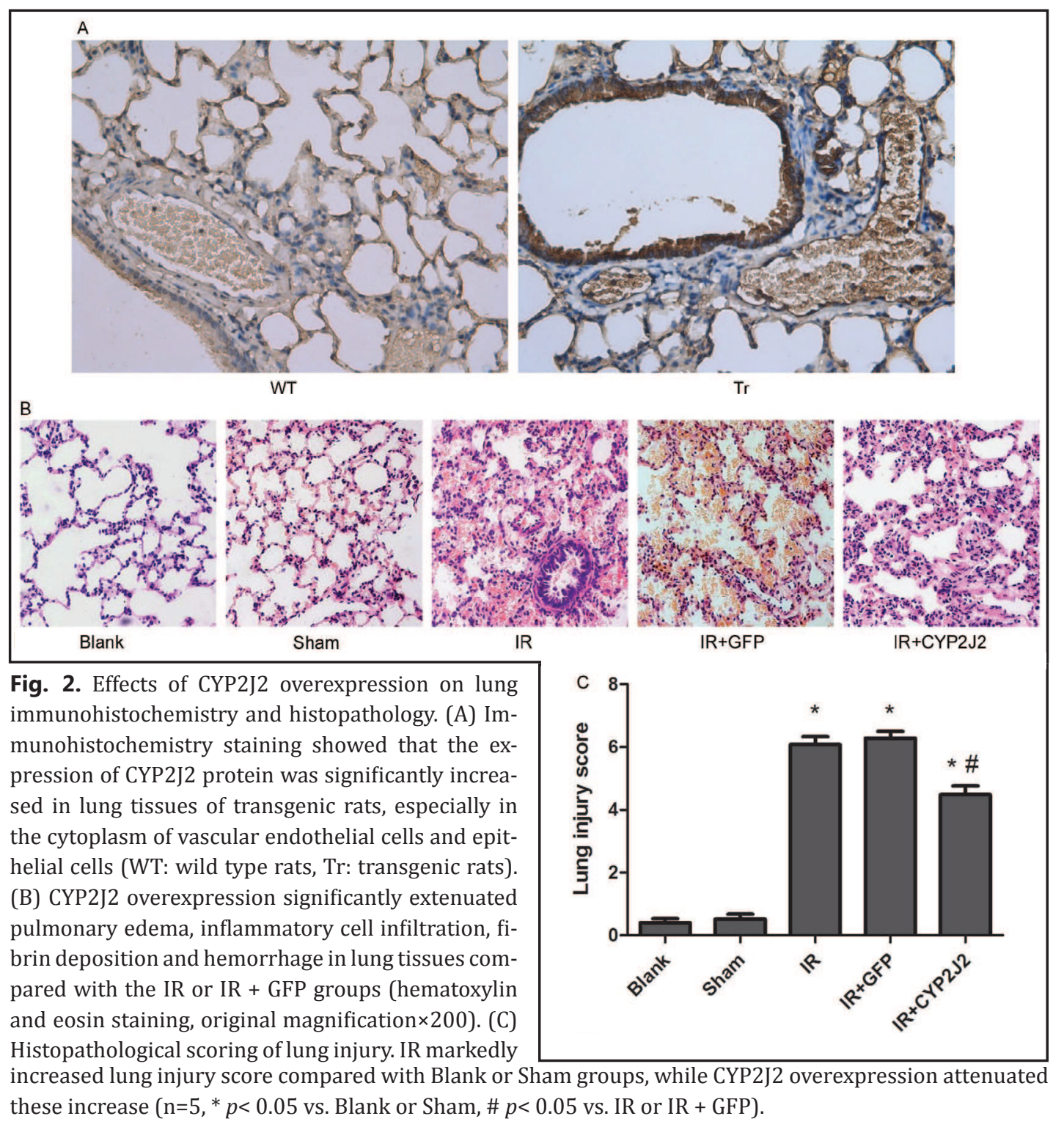

\section{Effect of CYP2J2 overexpression on protein concentration in BALF}

To assess vascular permeability, we detected the total protein concentration in BALF at the end of the reperfusion period. As shown in Fig. 4, the total protein concentration in BALF was markedly higher in IR groups than those in control groups without IR, while CYP2J2 overexpression inhibited the increase.

Effects of CYP2J2 overexpression on inflammatory mediators in serum and inflammationrelated protein expression in vivo

As depicted in Fig. 5A, IR notably increased serum levels of pro-inflammatory mediators, including IL-1 $\beta$, IL-8,TNF- $\alpha$, sP- and sE-selectin, but significantly decreased the serum level of IL-10, an anti-inflammatory cytokine. While, the changes were remarkably inhibited by CYP2J2 overexpression. Moreover, the levels of PPAR $\gamma$ and IкB $\alpha$ in the cytoplasm were markedly decreased after IR, while CYP2J2 overexpression attenuated the effect (Fig. 5B). Furthermore, expression of NF- $\kappa B$ in the nucleus after IR significantly increased compared to control groups without IR, an effect that was reversed by CYP2J2 overexpression (Fig. 5C). 


\section{Cellular Physiology \\ Cell Physiol Biochem 2015;35:2043-2054 and Biochemistry
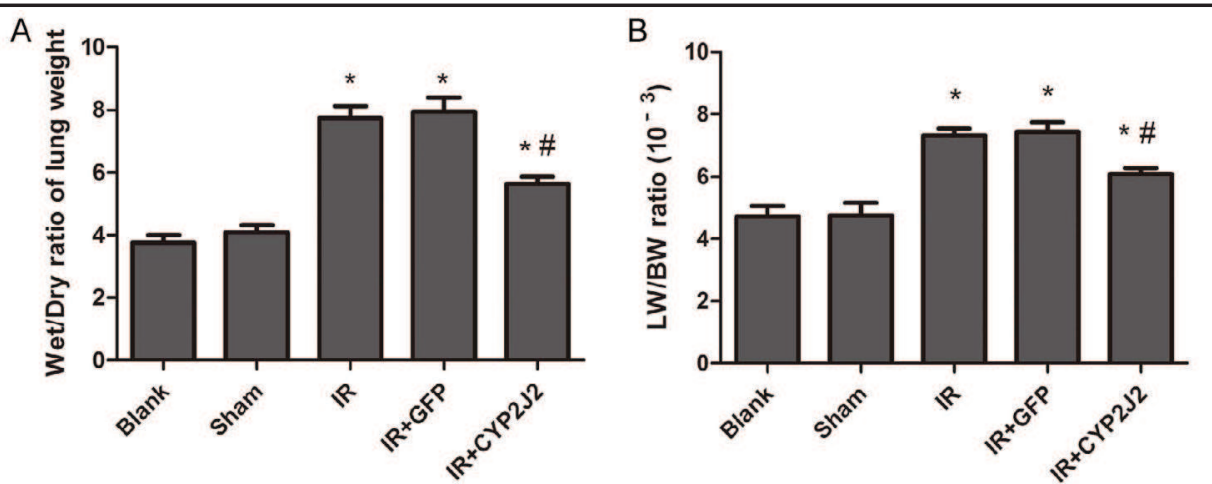

Fig. 3. Effects of CYP2J2 overexpression on lung W/D and LW/BW ratios. IR induced a significant increase in lung W/D (A) and LW/BW (B) ratios compared with control and Blank groups. While CYP2J2 overexpression reduced the increase after IR ( $\mathrm{n}=5, * p<0.05$ vs. Blank or Sham, \# $p<0.05$ vs. IR or IR + GFP).

Fig. 4. Effect of CYP2J2 overexpression on protein concentration in bronchoalveolar lavage fluid (BALF). IR notably increased the total protein concentration in BALF compared with Blank or Sham groups, an effect that was reversed by CYP2J2 overexpression ( $\mathrm{n}=4, * p<0.05$ vs. Blank or Sham, \# $p<$ 0.05 vs. IR or IR + GFP).

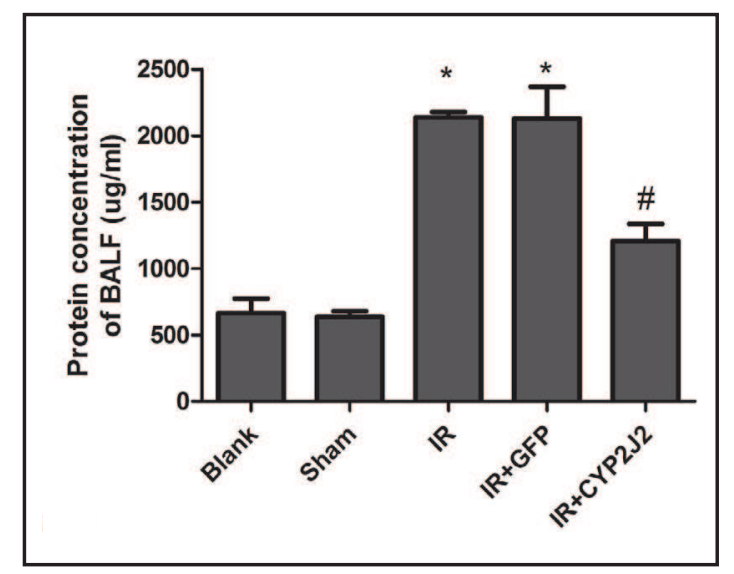

The anti-inflammatory effect of CYP2J2 overexpression and 11,12-EET is mediated by PPARY activation in HPAECS

As shown in Fig. 6A, the concentrations of pro-inflammatory cytokines in cell cultured media, including IL-1 $\beta$ and IL-6, were significantly increased after anoxia/reoxygenation compared with those in the control and vehicle groups. Interestingly, CYP2J2 overexpression remarkably attenuated these increases. However, these effects were notably reversed by GW9662, a selective inhibitor of PPAR $\gamma$ (Fig. 6A). In contrast, the level of IL-10 was reduced following anoxia/reoxygenation. CYP2J2 overexpression significantly attenuated the decrease, an effect that was inhibited by GW9662 (Fig. 6A). In addition, no significant difference was observed among AR groups in the concentrations of VCAM-1 and ICAM-1.

Consistent with observations in the in vivo lung IR model, anoxia/reoxygenation treatment markedly reduced PPAR $\gamma$ expression in HPAECs, and the effect was notably reversed by CYP2J2 overexpression and 11,12-EET pretreatment (Fig. 6B). Furthermore, anoxia/ reoxygenation treatment decreased the expression of $\mathrm{I} \kappa \mathrm{B} \alpha$ in the cytoplasm and led to a remarkable elevation in the nuclear translocation of NF- $\mathrm{kB}$ p65. As expected, pretreatment with CYP2J2 overexpression or 11,12-EET significantly suppressed anoxia/reoxygenationinduced NF- $\kappa$ B activation, an effect that was reversed by GW9662 (Fig. 6B and C).

\section{Discussion}

The results of the current study demonstrate that IR induces ALI, as characterized by increased lung W/D and LW/BW ratios, increased protein concentration of BALF and 


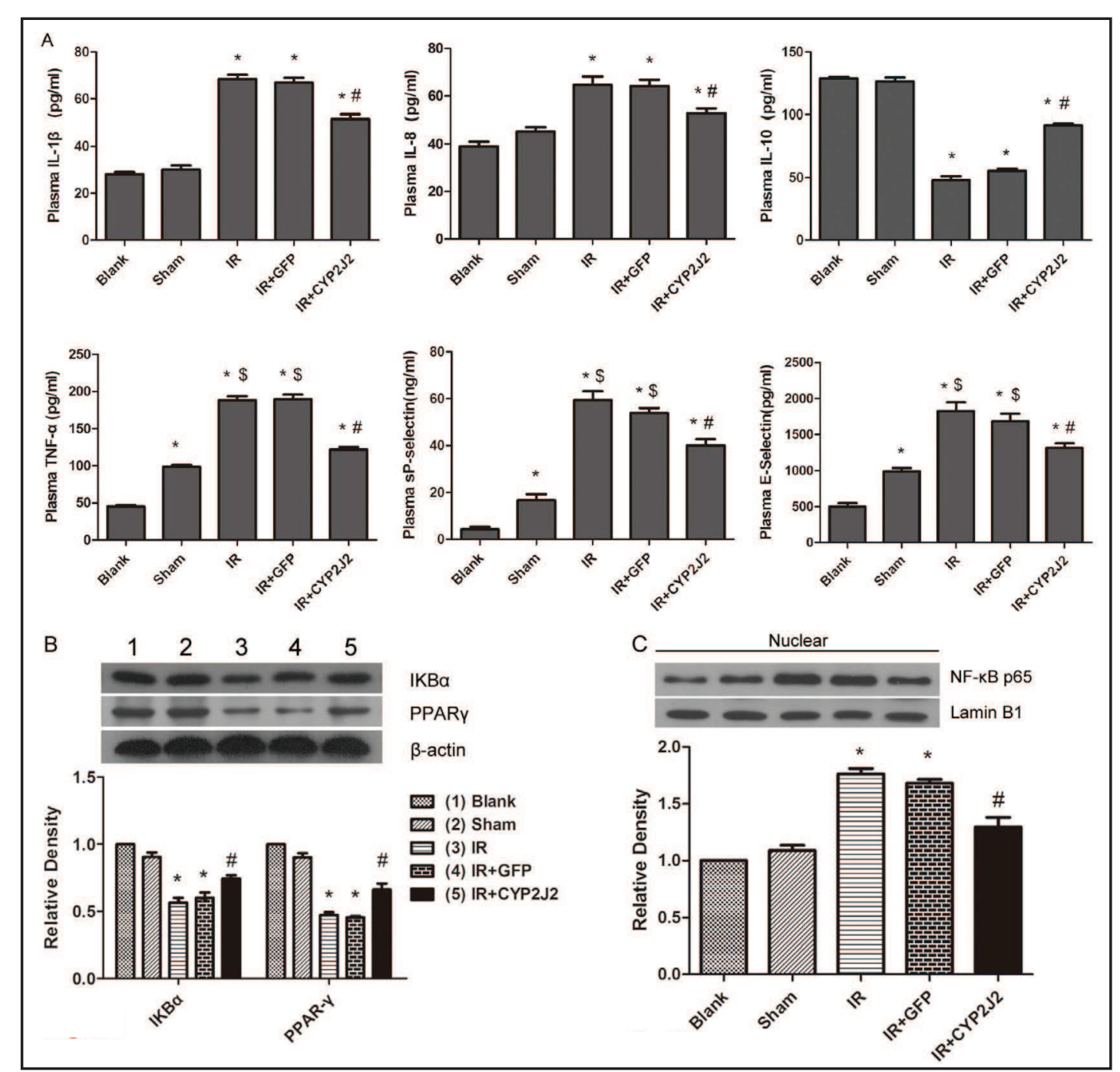

Fig. 5. Effects of CYP2J2 overexpression on inflammatory cytokines in serum and inflammation-related proteins in vivo. (A) Concentration of inflammatory cytokines in serum of rats ( $\mathrm{n}=10,{ }^{*} p<0.05$ vs. Blank, ${ }^{\$} p<$ 0.05 vs. Sham, $\# p<0.05$ vs. IR or IR + GFP). (B) Representative Western Blots and densitometry results indicated that CYP2J2 overexpression significantly inhibited IR-induced decrease of cytosolic IKB $\alpha$ and PPAR $\gamma$ expression in lung tissues. (C) Representative Western Blots and densitometry results showed that CYP2J2 overexpression remarkably attenuated IR-induced increase of nuclear NF- $\kappa$ B p65 expression in lung tissues $\left(\mathrm{n}=3,{ }^{*} p<0.05\right.$ vs. Blank or Sham, $\# p<0.05$ vs. IR or IR + GFP).

concentrations of pro-inflammatory mediators in serum, and decreased concentration of IL10 , an anti-inflammatory cytokine. Lung histopathology also revealed pulmonary edema and inflammation after IR. In addition, Western Blot demonstrated activation of NF- $\mathrm{BB}$ p65 and degradation of IKB $\alpha$ in IR lung tissues. Interestingly, immunohistochemical staining showed that CYP2J2 protein expression was significantly increased in lung tissues of transgenic rats, especially in the cytoplasm of vascular endothelial cells. In addition, CYP2J2 gene delivery increased circulating levels of EETs, attenuated lung injury following IR, and markedly improved pathohistological appearance. We further found that CYP2J2 overexpression and exogenous 11,12-EET treatment attenuated increases in pro-inflammatory cytokines following anoxia/reoxygenation in HPACEs. The protective effects may be mediated by activation of PPAR $\gamma$ and inhibition of NF- $\mathrm{KB}$ p65 nuclear translocation.

A wide variety of animal models have been developed to investigate the mechanisms and potential treatment of LIRI. In this study, we applied a consistent and reproducible rat 


\section{Cellular Physiology Cell Physiol Biochem 2015;35:2043-2054 \begin{tabular}{l|l} 
DOI: 10.1159/000374011 & O 2015 S. Karger AG, Basel
\end{tabular} and Biochemistry Publisned on Ine: vvarch 30,2015 www.karger.com/cpb}

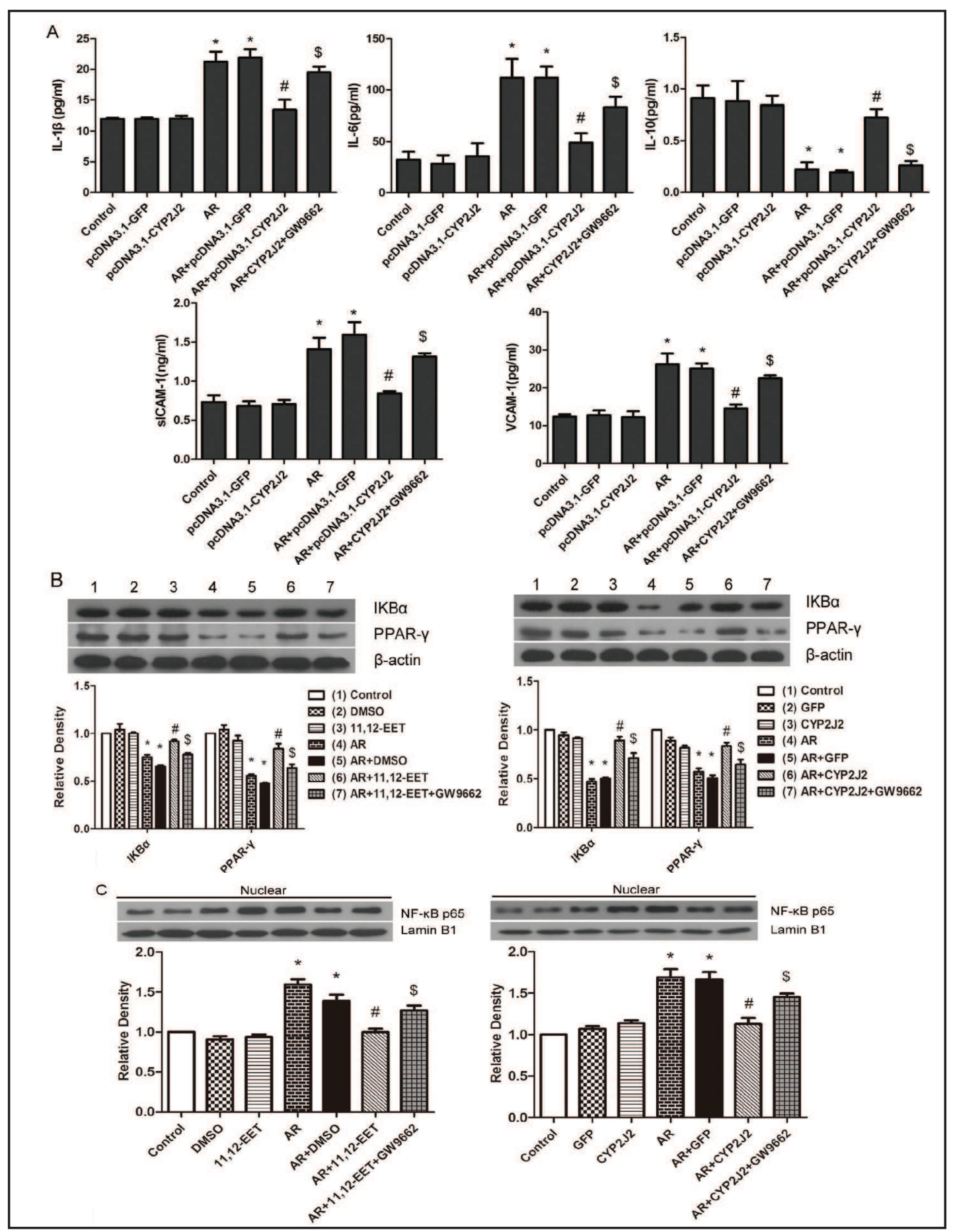

Fig. 6. The anti-inflammatory effect of CYP2J2 overexpression and 11,12-EET is mediated by PPAR $\gamma$ activation in HPAEC. (A) Concentration of inflammatory cytokines in cell cultured media of HPAECs $\left(\mathrm{n}=3, *{ }^{*} p<0.05\right.$ vs. control, \# $p<0.05$ vs. AR, ${ }^{\$} p<0.05$ vs. AR + CYP2J2). (B) Representative Western Blots and densitometry results indicated that CYP2J2 overexpression and exogenous 11,12-EET administration remarkably attenuated AR-induced decrease of cytosolic I $\mathrm{B} \alpha$ and PPAR $\gamma$ expression in HPAECs, an effect that was reversed by GW9662, a selective inhibitor of PPAR $\gamma$. (C) Representative Western Blots and densitometry results indicated that CYP2J2 overexpression and exogenous 11,12-EET treatment significantly attenuated increased levels of nuclear NF- $\mathrm{KB}$ p65 in HPAECs, while GW9662 administration blocked the effect $\left(\mathrm{n}=3,{ }^{*} p<0.05\right.$ vs. control, \# $p<0.05$ vs. AR, ${ }^{\$} p<0.05$ vs. AR + CYP2J2).

\section{KARGER}




\section{Cellular Physiology Cell Physiol Biochem 2015;35:2043-2054 \begin{tabular}{l|l} 
and Biochemistry Publisned onine: IVIarch 30, 2015 & $\begin{array}{l}\text { DOI 10.1159/00037011 } 2015 \text { S. Karger AG, Basel } \\
\text { www.karger.com/cpb }\end{array}$ \\
\hline
\end{tabular} \\ Chen et al.: EETs and Lung Ischemia/Reperfusion Injury}

model to explore the effect and mechanism of CYP2J2 overexpression on LIRI by clamping the pulmonary hilum. This is a widely used model which results in complete ischemia and anoxia [1]. It has been well documented that CYP2J2 is of human origin and is abundantly expressed in vascular endothelial cells [4]. Moreover, pulmonary vascular endothelial cell is an important part of the blood-air barrier to prevent movement of fluids and proteins into bronchoalveolar space and lung interstitium [13]. We therefore developed a HPAEC-based IR model in vitro.

Inflammatory responses are reported to play a pivotal role during LIRI, and be associated with multiple factors, including neutrophil activation, release of pro-inflammatory cytokines, and upregulation of molecules on the cell surface membrane [1,3]. In our study, increased pulmonary edema was observed in IR groups owing to increased permeability of the alveolarcapillary barrier. Microscopically, pulmonary edema, endothelial and epithelial injury are usually accompanied by neutrophil infiltration in the interstitium and bronchoalveolar space [13]. Activation and recruitment of neutrophils are considered to play a key role during the development of ALI [21]. Either inhibition of leukocyte adherence or neutrophil depletion reduces pulmonary vascular injury during IR [13]. In the current study, treatment with CYP2J2 overexpression remarkably attenuated inflammatory infiltration and hemorrhage in IR lung tissue, suggesting a protective effect of CYP2J2 via modulating neutrophil function.

Release of inflammatory cytokines plays an important role throughout the progression of LIRI. IL-8, which is known to stimulate neutrophil migration and activation, has been shown to dramatically increase after reperfusion [22]. Moreover, IL-8 level in lung tissue is directly related to mortality after human lung transplantation [23]. TNF- $\alpha$ and IL-1 $\beta$ are also upregulated during lung IR [1], as shown in our study. The levels of these cytokines are correlated with the degree of early pulmonary microvascular permeability, neutrophil sequestration, and lung edema, indicating that they are important modulators in the initial phase of LIRI $[1,24]$. In addition, the elevated levels of TNF- $\alpha$ and IL-1 $\beta$ contribute to neutrophil chemotaxis and production of other cytokines, resulting in late-phase injury [25]. In contrast, IL-10 exerts protective effect against LIRI via inhibition of inflammatory and T-cell-mediated immune responses [1]. It is suggested that IL-10 contributes to decreased levels of TNF- $\alpha$, IL-6, and reactive oxygen species[26]. In the current study, the expression of IL-1 $\beta$, IL-6, IL-8, TNF- $\alpha$ significantly increased in IR and AR groups compared to the control or vehicle groups. However, CYP2J2 overexpression notably decreased the concentrations of these pro-inflammatory cytokines. While, the opposite changes in IL-10 level were observed in our study. Thus, the beneficial effect of CYP2J2 overexpression may be partially due to regulating the production of inflammatory cytokines in IR-induced ALI.

Adhesion molecules have been reported to upregulate on pulmonary vascular endothelial cells during lung IR and promote leukocyte rolling [3]. While, blockade of adhesion molecules at the time of reperfusion can alleviate LIRI [3]. Our study demonstrated that the concentrations of sE-selectin and sP-selectin increased in the IR groups, an effect that was reversed by CYP2J2 overexpression. However, CYP2J2 overexpression did not decrease the concentrations of VCAM-1 and ICAM- 1 after AR. Further research is needed to explain the phenomenon.

$\mathrm{NF}-\mathrm{\kappa B}$ is considered to be one of the most important mediators throughout the whole process of LIRI and plays a central role in the induction of lung inflammatory injury by regulating the levels of inflammatory cytokines, chemokines, apoptotic signals, and cell adhesion molecules $[1,22,27]$. Inhibition of NF- $\mathrm{KB}$ activation reduces LIRI and improves pulmonary graft function in a porcine model [28]. The activation of PPAR $\gamma$ has been shown to suppress the activation of NF-кB [14]. In addition, EETs activate PPAR $\gamma$ [29]. It has also been reported that PPAR $\gamma$ pathway plays an important role in mediating the anti-inflammatory effects of CYP2J2 overexpression and EETs in VSMCs by suppressing NF- kB activation [5]. As observed in our study, IR significantly promoted the degradation of cytosolic I $\kappa \mathrm{B} \alpha$ and nuclear translocation of NF-KB p65 in vivo, an effect that was reversed by CYP2J2 overexpression. Meanwhile, PPAR $\gamma$ activity decreased in IR rat lungs and CYP2J2 overexpression attenuated the decrease. In vitro experiments further confirmed the above effects, and found that these 


\section{Cellular Physiology Cell Physiol Biochem 2015;35:2043-2054 \begin{tabular}{l|l} 
and Biochemistry Publisned onIne: IVIarch 30, 2015 & $\begin{array}{l}\text { C 2015 S. Karger AG, Basel } \\
\text { www.karger.com/cpb }\end{array}$ \\
\hline
\end{tabular}}

Chen et al.: EETs and Lung Ischemia/Reperfusion Injury

effects could be suppressed by GW9662, a selective PPAR $\gamma$ inhibitor, suggesting that the protective effect of CYP2J2 overexpression and exogenous 11,12-EET may be mediated by PPAR $\gamma$ activation.

In this preliminarystudy, we explored the effect and mechanism ofCYP2J2 overexpression and 11,12-EET in IR-induced ALI. The long-term effect of CYP2J2 overexpression and 11,12EET administration requires further study. Multiple factors and mechanisms are involved in the inflammatory process. The current study evaluated a few inflammatory mediators, and discussed the PPAR $\gamma /$ NF- $\mathrm{KB}$ pathway. Many pathophysiological changes during LIRI remain elusive. Further studies are still needed to provide a better understanding of this complicated protective mechanism.

In conclusion, the results of the current study indicate that CYP2J2 overexpression and 11,12-EET attenuate LIRI in vivo and in vitro via anti-inflammatory process. The protective effect may potentially be mediated by activation of PPAR $\gamma$. Thus, CYP2J2 gene delivery or EETs administration may be a potential preventive and therapeutic strategy against LIRI. Further investigations are required to confirm these findings.

\section{Disclosure Statement}

The authors have no conflict of interests to declare.

\section{Acknowledgments}

This work was supported by a National Natural Science Foundation of China Grant (No. 81000031).

\section{References}

1 den Hengst WA, Gielis JF, Lin JY, Van Schil PE, De Windt LJ, Moens AL: Lung ischemia-reperfusion injury: a molecular and clinical view on a complex pathophysiological process. Am J Physiol-Heart Circul Physiol 2010;299:H1283-H1299.

2 Ansari B, Laffey JG: Ischemia-reperfusion-induced lung injury: Could hypercapnia have a therapeutic role? Crit Care Med 2012;40:688-690.

-3 de Perrot M, Liu M, Waddell TK, Keshavjee S: Ischemia-reperfusion-induced lung injury. Am J Respir Crit Care Med 2003;167:490-511.

4 Node K, Huo Y, Ruan X, Yang B, Spiecker M, Ley K, Zeldin DC, Liao JK: Anti-inflammatory properties of cytochrome P450 epoxygenase-derived eicosanoids. Science 1999;285:1276-1279.

5 Cai Z, Zhao G, Yan J, Liu W, Feng W, Ma B, Yang L, Wang JA, Tu L, Wang DW: CYP2J2 overexpression increases EETs and protects against angiotensin II-induced abdominal aortic aneurysm in mice. J Lipid Res 2013;54:1448-1456.

6 Zhao G, Wang J, Xu X, Jing Y, Tu L, Li X, Chen C, Cianflone K, Wang P, Dackor RT, Zeldin DC, Wang DW: Epoxyeicosatrienoic acids protect rat hearts against tumor necrosis factor-alpha-induced injury. J Lipid Res 2012;53:456-466.

7 Zhao G, Tu L, Li X, Yang S, Chen C, Xu X, Wang P, Wang DW: Delivery of AAV2-CYP2J2 protects remnant kidney in the 5/6-nephrectomized rat via inhibition of apoptosis and fibrosis. Hum Gene Ther 2012;23:688-699.

8 Chen C, Wei X, Rao X, Wu J, Yang S, Chen F, Ma D, Zhou J, Dackor RT, Zeldin DC, Wang DW: Cytochrome P450 2J2 is highly expressed in hematologic malignant diseases and promotes tumor cell growth. J Pharmacol Exp Ther 2011;336:344-355.

-9 Li R, Xu X, Chen C, Yu X, Edin ML, Degraff LM, Lee CR, Zeldin DC, Wang DW: Cytochrome P450 2J2 is protective against global cerebral ischemia in transgenic mice. Prostaglandins Other Lipid Mediat 2012;99:68-78. 


\section{Cellular Physiology Cell Physiol Biochem 2015;35:2043-2054

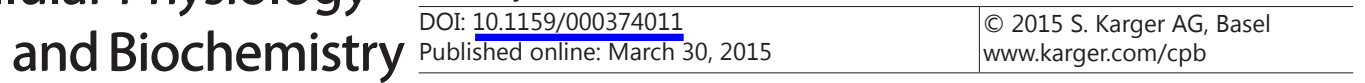

Chen et al.: EETs and Lung Ischemia/Reperfusion Injury

10 Seubert JM, Zeldin DC, Nithipatikom K, Gross GJ: Role of epoxyeicosatrienoic acids in protecting the myocardium following ischemia/reperfusion injury. Prostaglandins Other Lipid Mediat 2007;82:50-59.

11 Chen W, Zheng G, Yang S, Ping W, Fu X, Zhang N, Wang D, Wang J: CYP2J2 and EETs Protect against Oxidative Stress and Apoptosis in Vivo and in Vitro Following Lung Ischemia/Reperfusion. Cell Physiol Biochem2014;33:1663-1680.

12 Luce JM: Acute lung injury and the acute respiratory distress syndrome. Crit Care Med 1998;26:369-376.

13 Peng C-K, Huang K-L, Wu C-P, Li M-H, Hu Y-T, Hsu C-W, Tsai S-H, Chu S-J: Glutamine protects ischemiareperfusion induced acute lung injury in isolated rat lungs. Pulmon Pharmacol Ther 2011;24:153-161.

14 Appel S, Mirakaj V, Bringmann A, Weck MM, Grünebach F, Brossart P: PPAR- $\gamma$ agonists inhibit tolllike receptor-mediated activation of dendritic cells via the MAP kinase and NF- $\mathrm{KB}$ pathways. Blood 2005;106:3888-3894.

15 Ping W, Jiang WY, Chen WS, Sun W, Fu XN: Expression and significance of hypoxia inducible factor-1alpha and lysyl oxidase in non-small cell lung cancer. Asian Pac J Cancer Prev 2013;14:3613-3618.

-16 Patel B, Gupta N, Ahsan F: Low-Molecular-Weight Heparin (LMWH)-Loaded Large Porous PEG-PLGA Particles for the Treatment of Asthma. J Aerosol Med Pulm Drug Deliv 2014;27:12-20.

17 Yu T-C, Yang F-L, Hsu B-G, Wu W-T, Chen S-C, Lee R-P, Subeq Y-M: Deleterious effects of aggressive rapid crystalloid resuscitation on treatment of hyperinflammatory response and lung injury induced by hemorrhage in aging rats. J Surg Res 2014;187:587-595.

-18 Lindenberger M, Lanne T: Reduced defense of central blood volume during acute lower body negative pressure-induced hypovolemic circulatory stress in aging women. Shock 2012;37:579-585.

19 Lee CR, Imig JD, Edin ML, Foley J, DeGraff LM, Bradbury JA, Graves JP, Lih FB, Clark J, Myers P: Endothelial expression of human cytochrome $\mathrm{P} 450$ epoxygenases lowers blood pressure and attenuates hypertensioninduced renal injury in mice. FASEB Journal 2010;24:3770-3781.

20 Jiang J-G, Chen C-L, Card JW, Yang S, Chen J-X, Fu X-N, Ning Y-G, Xiao X, Zeldin DC, Wang DW: Cytochrome P450 2J2 promotes the neoplastic phenotype of carcinoma cells and is up-regulated in human tumors. Cancer Res 2005;65:4707-4715.

21 Grommes J, Soehnlein 0: Contribution of neutrophils to acute lung injury. Mol Med 2011;17:293.

22 Krishnadasan B, Naidu BV, Byrne K, Fraga C, Verrier ED, Mulligan MS: The role of proinflammatory cytokines in lung ischemia-reperfusion injury. J Thorac Cardiovasc Surg 2003;125:261-272.

23 De Perrot M, Sekine Y, Fischer S, Waddell TK, McRAE K, Liu M, Wigle DA, Keshavjee S: Interleukin-8 release during early reperfusion predicts graft function in human lung transplantation. Am J Respir Crit Care Med 2002;165:211-215.

24 Khimenko PL, Bagby G, Fuseler J, Taylor AE: Tumor necrosis factor- $\alpha$ in ischemia and reperfusion injury in rat lungs. Journal of Applied Physiology 1998;85:2005-2011.

-25 Goodman RB, Pugin J, Lee JS, Matthay MA: Cytokine-mediated inflammation in acute lung injury. Cytokine Growth Factor Rev 2003;14:523-535.

26 Eltzschig HK, Eckle T: Ischemia and reperfusion--from mechanism to translation. Nat Med 2011;17:13911401.

27 Liu SF, Malik AB: NF-kappa B activation as a pathological mechanism of septic shock and inflammation. Am J Physiol Lung Cell Mol Physiol 2006;290:L622-L645.

28 Ross SD, Kron IL, Gangemi JJ, Shockey KS, Stoler M, Kern JA, Tribble CG, Laubach VE: Attenuation of lung reperfusion injury after transplantation using an inhibitor of nuclear factor-kappaB. Am J Physiol Lung Cell Mol Physiol 2000;279:L528-536.

29 Liu Y, Zhang Y, Schmelzer K, Lee TS, Fang X, Zhu Y, Spector AA, Gill S, Morisseau C, Hammock BD, Shyy JY: The antiinflammatory effect of laminar flow: the role of PPARgamma, epoxyeicosatrienoic acids, and soluble epoxide hydrolase. Proc Natl Acad Sci U S A 2005;102:16747-16752. 